\title{
The Observer Interpretation Evolution and Collapse Determination in a Single 2-D Space
}

\author{
Yehuda Gavriel Roth \\ Oranim College, Oranim, Israel \\ Email:yudroth@gmail.com
}

Received October 16, 2013; revised November 16, 2013; accepted November 22, 2013

Copyright (C) 2014 Yehuda Gavriel Roth. This is an open access article distributed under the Creative Commons Attribution License, which permits unrestricted use, distribution, and reproduction in any medium, provided the original work is properly cited. In accordance of the Creative Commons Attribution License all Copyrights (C) 2014 are reserved for SCIRP and the owner of the intellectual property Yehuda Gavriel Roth. All Copyright (c) 2014 are guarded by law and by SCIRP as a guardian.

\begin{abstract}
We present a complete interpretation theory in the following sense: we observe that each measuring device represents a concept set (such as the set of locations) while the measurement activity associates the measured object with an appropriate member from the concepts set. In that sense, the measurement process is the only interpretation of reality. In this article, we deal with the evolution of this interpreting measuring device for a 2-D Hilbert space. It is shown that nonlinear recursive maps give rise to a unique projective operator accompanied with the collapse ability and consequently to a measuring device. Our formalism can be easily interpreted as a single brain signal.
\end{abstract}

\section{KEYWORDS}

\section{Interpretation; Complementary Maps}

\section{Introduction}

This paper follows other papers that assume a philosophical view that all we experienced or analyzed is an interpretation of reality rather than reviling some objective truth. For example, a pattern such as the one that appears in Figure 1 has two interpretations: the letter $\mathbf{B}$ or the number 13. Clearly giving some meaning to that vague pattern is only a brain interpretation [1,2]. In other papers, we proposed some mathematical formalism to understand the way we interpret reality. We used quantum ideas in the following manner: using the fact that many brain activities correspond with feedback loops (see for example ref. [3]) which are mostly associated with nonlinearity [3]. We refer this induced nonlinearity with a selective surrounding that simulates the brain activity through the nonlinearity of the recursive maps. We showed that nonlinear maps in the regular regime [4] can serve as a selective tool that filters only unique projective operators in which the observer can interpret reality. In this paper, we will show that nonlinear maps, now defined in the chaotic regime, give rise to the collapse phenomena. We note that although this collapse is defined with direct relation to the usual quantum collapse theories [5,6] it can also be understood purely in a mathematical sense. Therefore our interpretation theory does not necessarily define our theory as a part of quantum mechanics. Labelfont $=$ bf.

The aim of this paper is to establish a complete interpretation theory. By integrating between the chaotic and the regular maps, we show the complete theory showing the evolution of a measuring device, namely, the final unique projective operator with the collapse ability. We derive our formalism for a 2-D Hilbert space that can be easily interpreted as a single brain signal [7,8].

\section{Review-Single Parameter Complementary Maps}

\subsection{The Regular Complementary Maps}

The set of complementary maps that is responsible for the measuring device evolution is defined as: 
Figure 1. An example of the brain interpretation: This vague pattern can have interpretations: The letter $B$ or the number 13.

$$
\rho_{n+1}=\mathcal{R}\left(\rho_{n}\right), \rho \in[0,1] ; \bar{\rho}_{n+1}=1-\mathcal{R}\left(1-\bar{\rho}_{n}\right), \bar{\rho} \in[0,1]
$$

where $\mathcal{R}$ is a regular map function that determine the iteration of the $\rho$-parameter ( $\rho$ stand for “ $\rho$ egular"). For example, the logistic map,

$$
\begin{aligned}
& \mathcal{R}=R \rho(1-\rho) \\
& \text { that is } \\
& \rho_{n+1}=R \rho_{n}\left(1-\rho_{n}\right) \quad 0 \leq R<3 \\
& \bar{\rho}_{n+1}=1-R \bar{\rho}_{n}\left(1-\bar{\rho}_{n}\right) .
\end{aligned}
$$

With $R$ the strength parameter that defines the iteration type.

\subsection{Review—The Chaotic Complementary Maps}

The set of complementary maps that is responsible for the collapse are defined as [4]:

$$
\begin{aligned}
& \chi_{n+1}=X\left(\chi_{n}\right), \chi \in[0,1] \\
& \bar{\chi}_{n+1}=1-X\left(1-\bar{\chi}_{n}\right), \bar{\chi} \in[0,1]
\end{aligned}
$$

where $X$ is a chaotic map function that determine the iteration of the $\chi$-parameter ( $\chi$ stand for the Greek word $\chi a ́ o \varsigma)$.

In general if we observe each complementary map's parameters such as $\rho$ and $\bar{\rho}$ each map of the two can evolve independently provided that the initial variables are independently selected. Otherwise, when the initial conditions are coordinated such that $\rho_{0}+\bar{\rho}_{0}=1$ or $\chi_{0}+\bar{\chi}_{0}=1$ the maps are considered to be initially unitary correlated. Nevertheless, In our model we impose no correlation between the chaotic and regular maps as defined in equations (1) and (3). It can be shown, that in a pure mathematical sense, an initially unitary correlated coefficients, namely, $\rho_{0}+\bar{\rho}_{0}=1$ or $\chi_{0}+\bar{\chi}_{0}=1$, conserves coherence during the maps iterations' that is $\forall n\left(\begin{array}{l}\rho_{n}=1-\bar{\rho}_{n} \\ \chi_{n}=1-\bar{\chi}_{n}\end{array}\right)$. However, although the regular maps coherence last forever, chaotic maps suffer from coherence violation due to the "butterfly effect", that is, high sensitivity to an accumulative small random errors [4].

\section{States Representation}

\subsection{Review-The Regular States Definition as a Concept Generator}

Various models and in particular the various Spin-Glass-Models [9] associate brain activity with interacting spins models. In general we adapt the approach that an electrical "pulse" existence or absence is associated with a 2-D Hilbert space, such as the spin model, refers to the $|1\rangle$ and $|\varnothing\rangle$, respectively.

Assume the initial states:

$$
|0\rangle_{\rho}=\sqrt{\rho_{0}}|1\rangle+\sqrt{\bar{\rho}_{0}}|\varnothing\rangle ; \quad|\tilde{0}\rangle_{\rho}=-\sqrt{\bar{\rho}_{0}}|1\rangle+\sqrt{\rho_{0}}|\varnothing\rangle
$$

where the initial maps $\rho_{0}$ and $\bar{\rho}_{0}$ are unitary correlated meaning that $\rho_{0}+\bar{\rho}_{0}=1$.

We assume that the states interact with an external surrounding that modifies the states coefficients in according to the recursive-complementary maps to generate the iterating states:

$$
|n\rangle_{\rho}=\sqrt{\rho_{n}}|1\rangle+\sqrt{\bar{\rho}_{n}}|\varnothing\rangle ; \quad|\tilde{n}\rangle_{\rho}=-\sqrt{\bar{\rho}_{n}}|1\rangle+\sqrt{\rho_{n}}|\varnothing\rangle
$$

with the recursive maps of Equation (1). 


\subsection{Review-Concept Generation [4]}

Recursive maps such as the logistic map have the tendency of reaching constant values, provided that they are not in the chaotic regimes. If the maps reach the single values $\rho_{\infty}$ and $\bar{\rho}_{\infty}$ we obtain that any initial basis of states terminates at the unique basis:

$$
|n \rightarrow \infty\rangle_{\rho}=\sqrt{\rho_{\infty}}|1\rangle+\sqrt{\bar{\rho}_{\infty}}|\varnothing\rangle ; \quad|\tilde{n} \rightarrow \infty\rangle_{\rho}=-\sqrt{\bar{\rho}_{\infty}}|1\rangle+\sqrt{\rho_{\infty}}|\varnothing\rangle
$$

This final basis of state defines the observer unique measuring device.

We demonstrate the toy model formalism with the logistic formula where

$$
\rho_{n+1}=R \rho_{n}\left(1-\rho_{n}\right) ; \quad \bar{\rho}_{n+1}=1-R \bar{\rho}_{n}\left(1-\bar{\rho}_{n}\right) .
$$

If $R<3$ the maps converge into the single values $\rho_{\infty}=\frac{R-1}{R}$ and $\bar{\rho}_{\infty}=\frac{1}{R}$. Consequently we obtain states that except for the $\rho_{n}=0,1$-values, always converge into the defined basis of states:

$$
|\infty\rangle_{R}=\sqrt{\frac{R-1}{R}}|1\rangle+\sqrt{\frac{1}{R}}|\varnothing\rangle ; \quad|\tilde{\infty}\rangle_{R}=-\sqrt{\frac{1}{R}}|1\rangle+\sqrt{\frac{R-1}{R}}|\varnothing\rangle
$$

This basis of states defines a specific measuring device that can be related to a concept. However, in practice a real interpretation corresponds with a collapse mechanism. Indeed it was shown that chaotic maps generate the collapse as to be reviewed now.

\subsection{Review-Chaotic Maps as the Collapse Generator [4]}

The iterated states induced by the chaotic maps are:

$$
|n\rangle_{\chi}=\sqrt{\chi_{n}}|1\rangle+\sqrt{\bar{\chi}_{n}}|\varnothing\rangle ; \quad|\tilde{n}\rangle_{\chi}=-\sqrt{\bar{\chi}_{n}}|1\rangle+\sqrt{\chi_{n}}|\varnothing\rangle .
$$

We parameterize the maps as follows

$$
\cos \left(\theta_{n}\right)=\sqrt{\bar{\chi}_{n}} ; \quad \sin \left(\theta_{n}\right)=\sqrt{\chi_{n}},
$$

to obtain

$$
|n\rangle_{\chi}=\cos \theta_{n}|1\rangle+\sin \theta_{n}|\varnothing\rangle ; \quad|\tilde{n}\rangle_{\chi}=-\cos \theta_{n}|\varnothing\rangle+\sin \theta_{n}|1\rangle .
$$

\subsection{The Stationary States Representation}

From Equation (9) we can compose the states:

$$
|-\rangle_{n_{\chi}}=\frac{1}{\sqrt{2}}\left(|n\rangle_{\chi}+\mathrm{i}|\tilde{n}\rangle_{\chi}\right) ; \quad|+\rangle_{n_{\chi}}=\frac{1}{\sqrt{2}}\left(|n\rangle_{\chi}-\mathrm{i}|\tilde{n}\rangle_{\chi}\right)
$$

or

$$
|+\rangle_{n_{\chi}}=\mathrm{e}^{\mathrm{i} \theta_{n}}|+\rangle ; \quad|-\rangle_{n_{\chi}}=\mathrm{e}^{-\mathrm{i} \theta_{n}}|-\rangle
$$

where

$$
|+\rangle=\frac{1}{\sqrt{2}}(|1\rangle-\mathrm{i}|\varnothing\rangle) ; \quad|-\rangle=\frac{1}{\sqrt{2}}(|1\rangle+\mathrm{i}|\varnothing\rangle)
$$

These states are regarded as stationary since the maps iterations are reflected only through a global phases $\mathrm{e}^{\mathrm{ti} \theta_{n}}$.

We can now generate new states by means of superposition:

$$
\left|\phi_{n}\right\rangle=A \mathrm{e}^{\mathrm{i} \theta_{n}}|+\rangle+B \mathrm{e}^{-\mathrm{i} \theta_{n}}|-\rangle ; \quad\left|\bar{\phi}_{n}\right\rangle=-B^{*} \mathrm{e}^{\mathrm{i} \theta_{n}}|+\rangle+A^{*} \mathrm{e}^{-\mathrm{i} \theta_{n}}|-\rangle ; \quad|A|^{2}+|B|^{2}=1
$$

where $A$ and $B$ are any arbitrary complex coefficients.

\subsection{Projecting Operators with Respect to Measuring Devices}

In our formalism the essence of the measuring device collapse phenomena is defined by the chaotic maps and it 
is represented by the stationary based maps basis $|+\rangle$ and $|-\rangle$. All other states in the 2-D space are represented by the states $\left|\phi_{n}\right\rangle,\left|\bar{\phi}_{n}\right\rangle$ which possess an arbitrary superposition coefficients $A$ and $B$.

In according to standard quantum mechanics, the two bases $|+\rangle,|-\rangle$ and $\left|\phi_{n}\right\rangle,\left|\bar{\phi}_{n}\right\rangle$ are associated with the projective operators:

The stationary projective operator

$$
\hat{P}_{ \pm}=\lambda_{+}|+\rangle\left\langle+\left|+\lambda_{-}\right|-\right\rangle\langle-|
$$

and

The $\phi$ projective operator

$$
\hat{P}_{\phi}=\lambda_{\phi}\left|\phi_{n}\right\rangle\left\langle\phi_{n}\left|++\lambda_{\bar{\phi}}\right| \bar{\phi}_{n}\right\rangle\left\langle\bar{\phi}_{n}\right|
$$

The projecting operator $\hat{P}_{\phi}$ presented in the stationary basis $| \pm\rangle$ becomes:

$$
\begin{aligned}
& \hat{P}_{\phi}=\hat{P}_{\phi}^{D}+\hat{P}_{\phi}^{I} \\
& \hat{P}_{\phi}^{D}=\lambda_{\phi}\left(|A|^{2}|+\rangle\left\langle+\left.|+| B\right|^{2} \mid-\right\rangle\langle-|\right)++\lambda_{\tilde{\phi}}\left(|B|^{2}|+\rangle\left\langle+\left.|+| A\right|^{2} \mid-\right\rangle\langle-|\right) \\
& \hat{P}_{\phi}^{I}=\left\{B^{*} A \mathrm{e}^{2 \mathrm{i} \theta_{n}}|+\rangle\left\langle-\left|+A^{*} B \mathrm{e}^{2 \mathrm{i} \theta_{n}}\right|-\right\rangle\langle+|\right\}\left(\lambda_{\phi}-\lambda_{\bar{\phi}}\right)
\end{aligned}
$$

The projective operator $\hat{P}_{\phi}$ as originally presented in the $\phi$-basis (Equation (17)) is a mathematical expression composed of the linear combination of $\phi$ and $\tilde{\phi}$-corresponding projective operators. An operator of the $\hat{P}_{\phi}$-type describes a measuring device that measures the $|\phi\rangle$-states (Equation (15)) with a complete certainty. However, it is incapable of describing a device that detects other bases. Indeed, when the same operator $\hat{P}_{\phi}$ is presented in the stationary basis $| \pm\rangle$ (Equation (18)), we obtained an interference term, $\hat{P}_{\phi}^{I}$, which is no longer composed of the linear combination of projective operators. States mixing such as $|-\rangle\langle+|$ as appeared in the interference term causes the whole expression to become inappropriate in describing a measuring device.

Although the interference part causes the expression of Equation (18) to be inappropriate in describing a measuring device, the first term $\hat{P}_{\phi}^{D}$ can certainly present a measuring device provided it is accompanied with statistical characteristics. The measurement reading $\lambda_{\phi}$ indicates the possibility that the device can detect the $|\phi\rangle_{n}$ state. Indeed for our state- $|\phi\rangle_{n}=A \mathrm{e}^{\mathrm{i} \theta_{n}}|+\rangle+B \mathrm{e}^{-\mathrm{i} \theta_{n}}|-\rangle$-we obtain that the expressions $|A|^{2}$ and $|B|^{2}$ that accompany the states $|+\rangle_{n}$ and $|-\rangle_{n}$ can be interpreted as the probabilities of detecting the appropriate states in agreement with the Born interpretation. Detecting the other eigenvalue $\lambda_{\tilde{\phi}}$ also provides the appropriate stationary states probabilities.

To summarize: The projective operator $\hat{P}_{\phi}$ presented in the stationary basis is composed of two parts: The interference term, $\hat{P}_{\phi}^{I}$, that violates the possibility of identifying the operator with a measuring device and the diagonal part, $\hat{P}_{\phi}^{D}$ which in according to the Born interpretation can be associated with a measuring device. It will be shown that in the chaotic regime by randomizing the maps phase, the interference term will vanish, on the average, leaving us with an appropriate expression for a measuring device.

\subsection{Phase Randomization-Numeric Calculation}

The first step in transforming the $\hat{P}_{\phi}$-projective-operator into a measuring device is to eliminate the interference terms.

This common angle $\theta_{n}$ suggests that the maps states $|n\rangle$ and $|\bar{n}\rangle$ describe a single vector with the coordinates $|x\rangle$ and $|y\rangle$. This vector rotates during the iterations in according to the values of $\theta_{n}$. On the contrary, an uncorrelated $|n\rangle$ and $|\tilde{n}\rangle$-vectors would possess two independent angles $\theta_{x n}$ and $\theta_{y n}$, as follows:

$$
\sin \theta_{\chi^{n}} \equiv \sqrt{\chi_{n}} ; \quad \cos \theta_{\bar{\chi}^{n}} \equiv \sqrt{\bar{\chi}_{n}} .
$$

In a numeric analysis we processed the logistic maps that were subject to a coherent initial condition $\left(\chi_{0}+\bar{\chi}_{0}=1\right)$. At each iteration we calculated the angles $\theta_{\chi^{n}}+\theta_{0}$ and $\theta_{\bar{\chi} n}+\theta_{0}$ according to Equation (19) and we plotted a correlation graph of $\theta_{\chi^{n}}+\theta_{0}$ verses $\theta_{\bar{\chi} n}+\theta_{0}$. The results are demonstrated in Figure 2.

For $R=3.4$ (left side) the case for which the maps are in the regular regime and therefore coherent for all 

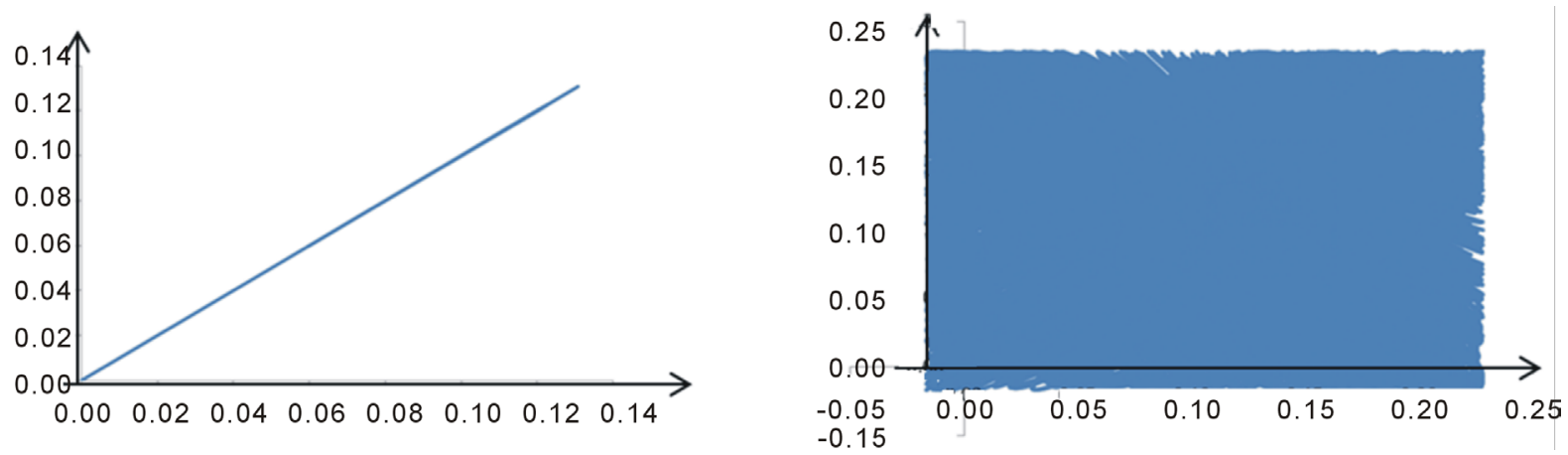

Figure 2. Numeric data for the graphs $\theta_{\chi^{n}}$ verses $\theta_{\chi^{n}}$ applied for the logistic maps with $\rho_{0}, \chi_{0}=0.7$ and $\bar{\rho}_{0}, \bar{\chi}_{0}=0.3$. On the left, the strength parameter is in the regular regime $-R=3.4$. The straight line demonstrates the correlation relation $\theta_{\rho n}=\theta_{\bar{\rho} n}$. On the left: The strength parameters is in the chaotic regime $(R=4):$ The discorrelation between the angles is represented by the point-filled-squares.

$n$, we obtained the straight line $\forall n \theta_{\rho n}=\tilde{\theta}_{\bar{\rho} n}$. The right graph shows the relations for $R=3.7$. The dis-correlation between the angles is represented by the point-filled-squares. Labelfont $=\mathrm{bf}$.

Clearly, during the iterations and long after the coherence time, the phase $\theta_{n}$ exhibit a random behavior.

Going back to the projecting operator as defined in Equation (17), calculating the time average of the projective operator, we obtain:

$$
\overline{\hat{P}}_{\phi_{n}}=\lambda_{\phi}|A|^{2}|+\rangle\left\langle+\left.\left|+\lambda_{\tilde{\phi}}\right| B\right|^{2} \mid-\right\rangle\langle-|
$$

We note that the eigenvalues $\lambda_{\phi}$ that are associated with the measurement readings are defined to be independent of the index $n$. Otherwise, we will obtain different readings for the same stationary state.

In conclusion, all the measuring environment effects which is reflected through the chaotic maps, provides us with the ability of formulating a mathematical frame to analyze the collapse phenomenon where in the present section we demonstrated our formalism by numerical means. In the following part we show that the phase randomization can be obtained with the Lyapunov exponent [8]. We note that although quantum collapse is discussed in refs [10-13], our formalism can be applied in classical systems.

\section{Maps Integration, the Complete Evolution of a Concept}

The chaotic and regular maps control the concept determination. During the coherence time which is determined by the chaotic maps, the regular maps cause the basis of states to stabilized at a single set. Long after the coherence time, deep in the chaotic stage the selected projective operator becomes a collapsing tool.

The composed states are

$$
|n\rangle_{\rho, \chi}=\sqrt{\rho_{n}} \mathrm{e}^{\mathrm{i} \theta_{n}}|+\rangle+\sqrt{\bar{\rho}_{n}} \mathrm{e}^{-\mathrm{i} \theta_{n}}|-\rangle ; \quad|\tilde{n}\rangle_{\rho, \chi}=-\sqrt{\bar{\rho}_{n}} \mathrm{e}^{\mathrm{i} \theta_{n}}|+\rangle+\sqrt{\rho_{n}} \mathrm{e}^{-\mathrm{i} \theta_{n}}|-\rangle
$$

where the $\rho$-variable iterates in according to the regular $\mathrm{R}$ generating function while the phases $\theta$ is determined by the chaotic $X$ function.

We assume that the states converge into a final basis in the coherent time period. Thus for $t<t_{c}$ we have the final regular basis:

$$
\left|\phi_{n}\right\rangle_{\rho, \chi}=\sqrt{\rho_{\infty}} \mathrm{e}^{\mathrm{i} \theta_{n}}|+\rangle+\sqrt{\bar{\rho}_{\infty}}|\rangle \mathrm{e}^{-\mathrm{i} \theta_{n}}|-\rangle ; \quad\left|\tilde{\phi}_{n}\right\rangle_{\rho, \chi}=-\sqrt{\bar{\rho}_{\infty}} \mathrm{e}^{\mathrm{i} \theta_{n}}|+\rangle+\sqrt{\rho_{\infty}}|\rangle \mathrm{e}^{-\mathrm{i} \theta_{n}}|-\rangle
$$

Equation (22) has the similar form of Equation (15) with $A \rightarrow \sqrt{\rho_{\infty}}$ and $B \rightarrow \sqrt{\bar{\rho}_{\infty}}$. Thus following the formalism we derived earlier we obtain that in according to eq. 20 the final projective operator is:

$$
\overline{\hat{P}}_{\phi_{n}}=\rho_{\infty} \lambda_{\phi}|+\rangle\left\langle+\left|+\bar{\rho}_{\infty} \lambda_{\tilde{\phi}}\right|-\right\rangle\langle-|
$$

Now, not only that we have a single selected projective operator, the mathematical expression can have an interpretation of a collapse mechanism. To be more specific, a measurement of any state can provide the values 
$\lambda_{\phi}$ or $\lambda_{\tilde{\phi}}$ that are associates with the $|\phi\rangle^{\prime}$ states with the probabilities $\rho_{\infty}$ and $\bar{\rho}_{\infty}$, respectively.

\section{Brain Activity in Terms of the Nonlinear-Maps}

Until now, we showed that the evolution of the non-linear recursive maps corresponded with an exclusive selection of preferable bases that span the Hilbert space. Each basis is associated with a measuring device for which, through measurements, the observer experiences and interprets the physical word.

The purpose of this part is to associate the brain activity with this 2-D model model.

Let us first briefly describe the brain activity by means of its electrical pulses: The brain is composed of nerve cells referred as neurons. All neurons in the brain are electrically excitable, maintaining voltage gradients across their membranes. Changes in the cross-membrane voltage can alter the function of voltage-dependent ion channels. If the voltage changes by a large enough amount, an all-or-none electrochemical pulse called an action potential or simply a signal, is generated. The signal travels along the cell's axon, and when it arrives to a junction it activates synaptic connections with other cells. We associate this brain activity with the nonlinear recursive maps where the sequential synapses activity is identified with the maps-parameter $n$.

In our model, the absence or excitation of a single is identified with the states $|\varnothing\rangle$ and $|1\rangle$, respectively.

Describing the brain activity by means of a 2-D Hilbert space was introduced long ago with the spin-glassmodels [9]. However, these spin glass models consist of a spin interaction Hamiltonian that determines the brain activity time evolution while our approach consists of a discrete time evolution determined by the non-linear recursive maps.

In our formalism, the transition between the ground and the excited states corresponds with the recursive regular and chaotic maps where the regular map is responsible for the concept definition while the chaotic maps enforce any measurement to fall between one of the measuring device concepts. Here we demonstrated our formalism for a single bite but we are convinced that it can be generalized to a multi-dimensional system.

\section{REFERENCES}

[1] Y. G. Roth, “Bifurcation and Pattern Recognition,” Journal of Modern Physics, Vol. 4, No. 1, 2013, pp. $25-29$. http://dx.doi.org/10.4236/jmp.2013.41005

[2] Y. G. Roth, “Single Measurement of Figures,” Journal of Modern Physics, Vol. 4, No. 6, 2013, pp. 812-817.

[3] S. S. Ge, C. C. Hang and T. Zhang, "Systems, Man, and Cybernetics, Part B,” Cybernetics, Vol. 29, No. 6, 1999 , pp. 818-828.

[4] Y. G. Roth, “The Evolution of Quantum Measuring Devices,” Accepted for Publication at the IC-MSQUARE Conference Proceedings Book, 2013.

[5] R. Penrose, “The Road to Reality: A Complete Guide to the Laws of the Universe, Ch. 2, Vintage Books,” 2004.

[6] G. C. Ghirardi, A. Rimini and T. Weber, "Unified Dynamics for Microscopic and Macroscopic Systems,” Physical Review D, Vol. 34, No. 2, 1986, pp. 470-491. http://dx.doi.org/10.1103/PhysRevD.34.470

[7] D. J. Amit and H. Gutfreund, "Spin-Glass Models of Neural Networks,” Physical Review A, Vol. 32, No. 2, 1985, pp. 10071018.

[8] A. Wolf, J. B. Swift, H. L. Swinney and J. A. Vastano, “Determining Lyapunov Exponents from a Time Series,” Physica D, Vol. 16, No. 3, 1985, pp. 285-317. http://dx.doi.org/10.1016/0167-2789(85)90011-9

[9] D. L. Stein and C. M. Newman, “Spin Glasses and Complexity (Primers in Complex Systems), Ch. 1,” Princeton University, Princeton, 2013.

[10] A. Bassi, "Dynamical Reduction Models: Present Status and Future Developments,” Journal of Physics: Conference Series, Vol. 67, 2007, Article ID: 012013.

[11] A. Bassi and D. G. M. Salvetti, “The Quantum Theory of Measurement within Dynamical Reduction Models,” Journal of Physics A: Mathematical Theory, Vol. 40, No. 32, 2007, p. 9859. http://dx.doi.org/10.1088/1751-8113/40/32/011

[12] W. H. Zurek, “Decoherence and the Transition from Quantum to Classical,” Physics Today, Vol. 44, No. 10, 1991, pp. 36-44. http://dx.doi.org/10.1063/1.881293

[13] S. L. Adler and A. Bassi, “Collapse Models with Non-White Noises,” Journal of Physics A: Mathematical Theory, Vol. 40, No. 50, 2007, pp. 15083-15098. http://dx.doi.org/10.1088/1751-8113/40/50/012 